\title{
Predicting another doubly charmed molecular resonance $\boldsymbol{T}_{c c}^{\prime+}(\mathbf{3 8 7 6})$
}

\author{
Rui Chen ${ }^{1,2, *}$ Qi Huang, ${ }^{3,6, \dagger}$ Xiang Liu $\odot,{ }^{4,5,6, *}$ and Shi-Lin Zhu $\odot^{2, \S}$ \\ ${ }^{1}$ Key Laboratory of Low-Dimensional Quantum Structures and Quantum Control of Ministry of Education, \\ Department of Physics and Synergetic Innovation Center for Quantum Effects and Applications, \\ Hunan Normal University, Changsha 410081, China \\ ${ }^{2}$ Center of High Energy Physics, Peking University, Beijing 100871, China \\ ${ }^{3}$ School of Physical Sciences, University of Chinese Academy of Sciences, Beijing 100049, China \\ ${ }^{4}$ School of Physical Science and Technology, Lanzhou University, Lanzhou 730000, China \\ ${ }^{5}$ Research Center for Hadron and CSR Physics, Lanzhou University \\ and Institute of Modern Physics of CAS, Lanzhou 730000, China \\ ${ }^{6}$ Lanzhou Center for Theoretical Physics, Key Laboratory of Theoretical Physics of Gansu Province, \\ and Frontiers Science Center for Rare Isotopes, Lanzhou University, Lanzhou 730000, China
}

(Received 11 August 2021; accepted 14 December 2021; published 30 December 2021)

The isospin breaking effect plays an essential role in generating hadronic molecular states with a very tiny binding energy. Very recently, the LHCb Collaboration observed a very narrow doubly charmed tetraquark $T_{c c}^{+}$in the $D^{0} D^{0} \pi$ mass spectrum, which lies just below the $D^{0} D^{*+}$ threshold around $273 \mathrm{keV}$. In this work, we study the $D^{0} D^{*+} / D^{+} D^{* 0}$ interactions with the one-bosonexchange effective potentials and consider the isospin breaking effect carefully. We not only reproduce the mass of the newly observed $T_{c c}^{+}$very well in the doubly charmed molecular tetraquark scenario, but also predict the other doubly charmed partner resonance $T_{c c}^{++}$with $m=3876 \mathrm{MeV}$, and $\Gamma=412 \mathrm{keV}$. The prime decay modes of the $T_{c c}^{+}$are $D^{0} D^{+} \gamma$ and $D^{+} D^{0} \pi^{0}$. The peculiar characteristic mass spectrum of the $D^{0} D^{*+} / D^{+} D^{* 0}$ molecular systems can be applied to identify the doubly charmed molecular states.

DOI: $10.1103 /$ PhysRevD.104.114042

\section{INTRODUCTION}

As an important and effective approach to shed light on the nonperturbative behavior of the quantum chromodynamics (QCD), the study of the hadron spectroscopy has become an active research field. Among abundant research issues around the hadron spectroscopy, searching for the exotic hadronic matter is full of challenges and opportunities at the birth of quark model [1-3]. There exist different exotic hadronic matters like glueball, hybrid, multiquark states.

Very recently, the LHCb Collaboration reported a very narrow state $T_{c c}^{+}$in the $D^{0} D^{0} \pi^{+}$mass spectrum with the significance over $10 \sigma$ [4,5]. It is the first observed doubly charmed tetraquark as its simplest valence quark

\footnotetext{
chenrui@hunnu.edu.cn

†huangqi@ucas.ac.cn

*xiangliu@lzu.edu.cn

§husl@pku.edu.cn
}

Published by the American Physical Society under the terms of the Creative Commons Attribution 4.0 International license. Further distribution of this work must maintain attribution to the author(s) and the published article's title, journal citation, and DOI. Funded by SCOAP. component is $c c \bar{u} \bar{d}$. Its spin-parity is assumed as $J^{P}=1^{+}$. Its mass with respect to the $D^{0} D^{*+}$ threshold and width are

$$
\begin{aligned}
\delta m & =m_{T_{c c}^{+}}-\left(m_{D^{0}}+m_{D^{*+}}\right)=-273 \pm 61 \pm 5_{-14}^{+11} \mathrm{keV} / c^{2}, \\
\Gamma & =410 \pm 165 \pm 43_{-38}^{+18} \mathrm{keV},
\end{aligned}
$$

respectively. This important observation shall push the exploration of exotic hadronic matter into a new era.

Since this doubly charmed tetraquark $T_{c c}^{+}$locates just below the $D D^{*}$ threshold, the doubly charmed mesonmeson molecule is the very attractive and probable explanation of the newly $T_{c c}^{+}$state. The predicted mass of the $D D^{*}$ molecule with $I\left(J^{P}\right)=0\left(1^{+}\right)[6,7]$ is very consistent with the mass of the newly $T_{c c}^{+}$. The binding energy is very small, which is similar to the case of the $X(3872)$ as an isoscalar $D \bar{D}^{*}$ molecule. The $T_{c c}^{+}$and $X(3872)$ shares the same one-pion-exchange force and nearly the same isospin breaking pattern. The very tiny binding energy can amplify the isospin breaking effect [8], which are also important for single heavy tetraquarks [9]. In fact, the interactions between the $D^{0} D^{*+}$ and $D^{+} D^{* 0}$ are almost the same. Therefore, if the $T_{c c}^{+}$is the $D^{0} D^{*+}$ molecule, there should 
exist the other $D^{+} D^{* 0}$ molecule just below the $D^{+} D^{* 0}$ threshold. When we recall the experimental information in the $D^{0} D^{0} \pi^{+}$mass spectrum, one can find a minor structure existing between the $D^{0} D^{*+}$ and $D^{+} D^{* 0}$ threshold, which may correspond to the $D^{+} D^{* 0}$ bound state.

In this work, we perform a coupled channel analysis of the $D^{0} D^{*+} / D^{+} D^{* 0}$ interactions by adopting the OBE effective potential and considering both the isospin breaking effect and $S-D$ wave mixing effect. We not only find that the newly $T_{c c}^{+}$state perfectly matches the very shallow bound $D^{0} D^{*+} / D^{+} D^{* 0}$ molecular explanation with $J^{P}=1^{+}$, but also obtain a coupled $T_{c c}^{\prime+}$ resonance with $m=3876 \mathrm{MeV}, \Gamma=412 \mathrm{keV}$. The $T_{c c}^{+}$molecular state is mainly composed of the $S$-wave $D^{0} D^{*+}$ component with over $70 \%$ probability. The dominant channel for the $T_{c c}^{+}$ resonance is the $S$-wave $D^{+} D^{* 0}$ component. The quantitative analysis on the strong decay behavior for the $T_{c c}^{\prime+}$ resonance indicates the $D^{+} D^{0} \pi^{0}$ channel is more important than the $D^{0} D^{0} \pi^{+}$, which explains why the experimental events for the $T_{c c}^{++}$in the $D^{0} D^{0} \pi^{+}$mass spectrum are not so significant. Nevertheless, it is likely to search for the $T_{c c}^{++}$state in the $D^{0} D^{+} \gamma$ and $D^{+} D^{0} \pi^{0}$ final states.

\section{II. $D^{0} D^{*+} / D^{+} D^{* 0}$ INTERACTIONS AND THE ISOSPIN BREAKING EFFECT}

In this work, a crucial input is the interaction between the charmed mesons. So far, the understanding of the realistic interactions is not enough due to the poor experimental data. Several phenomenological models have been put forward, such as the multiquark model, the QCD sum rule, the OBE model, the effective field theory (see reviews [10-12] for details. Especially the $T_{c c}$ has been reviewed extensively in Ref. [11]). We adopt the OBE model to study the mass spectrum of the $D^{0} D^{*+} / D^{+} D^{* 0}$ system with $J^{P}=$ $1^{+}$and consider the $\pi / \sigma / \eta / \rho / \omega$ meson exchange interactions.

The general procedure for deducing the OBE effective potentials is organized as follows. After constructing the effective Lagrangians, one can easily write down the OBE scattering amplitude $\mathcal{M}\left[M_{1} M_{2} \rightarrow M_{3} M_{4}\right]$ for the $M_{1} M_{2} \rightarrow$ $M_{3} M_{4}$ process. The OBE effective potentials in the momentum space can be related to the corresponding scattering amplitudes by a Breit approximation, $\mathcal{V}(\boldsymbol{q})=-\mathcal{M}\left[M_{1} M_{2} \rightarrow M_{3} M_{4}\right] / \sqrt{16 m_{M_{1}} m_{M_{2}} m_{M_{3}} m_{M_{4}}}$. In order to obtain the OBE effective potentials in the coordinate space, we further perform a Fourier transformation, $\mathcal{V}(\boldsymbol{r})=\int \frac{d^{3} \boldsymbol{q} e^{i q \cdot \boldsymbol{r}}}{(2 \pi)^{3}} \mathcal{V}(\boldsymbol{q}) \mathcal{F}^{2}\left(q^{2}, m_{E}^{2}\right)$. Here, we introduce a monopole form factor at every interaction vertices to compensate the off-shell effect of the exchanged meson, which has the form of $\mathcal{F}^{2}\left(q^{2}, m_{E}^{2}\right)=\left(\Lambda^{2}-m_{E}^{2}\right) /\left(q^{2}-m_{E}^{2}\right)$. $\Lambda, m_{E}$, and $q$ stand for the cutoff, mass and four-momentum of the exchanged particle, respectively.
The relevant effective Lagrangians to describe the interactions between the $S$-wave charmed mesons and the light mesons are constructed in terms of heavy quark symmetry and chiral symmetry [13-18], i.e.,

$$
\begin{gathered}
\mathcal{L}_{\mathcal{P}^{(*)} \mathcal{P}^{(*)} \sigma}=-2 g_{s} \mathcal{P}_{b}^{\dagger} \mathcal{P}_{b} \sigma-2 g_{s} \mathcal{P}_{b}^{*} \cdot \mathcal{P}_{b}^{* \dagger} \sigma \\
\mathcal{L}_{\mathcal{P}^{(*)} \mathcal{P}^{(*)} \mathbb{P}}=-\frac{2 g}{f_{\pi}}\left(\mathcal{P}_{b} \mathcal{P}_{a \lambda}^{* \dagger}+\mathcal{P}_{b \lambda}^{*} \mathcal{P}_{a}^{\dagger}\right) \partial^{\lambda} \mathbb{P}_{b a} \\
-i \frac{2 g}{f_{\pi}} v^{\alpha} \varepsilon_{\alpha \mu \nu \lambda} \mathcal{P}_{b}^{* \mu} \mathcal{P}_{a}^{* \lambda \dagger} \partial^{\nu} \mathbb{P}_{b a}, \\
\mathcal{L}_{\mathcal{P}^{(*)} \mathcal{P}^{(*)} \mathbb{V}=}-\sqrt{2} \beta g_{V} \mathcal{P}_{b} \cdot \mathcal{P}_{a}^{\dagger} v \cdot \mathbb{V}_{b a} \\
-2 \sqrt{2} \lambda g_{V} v^{\lambda} \varepsilon_{\lambda \mu \alpha \beta}\left(\mathcal{P}_{b} \mathcal{P}_{a}^{* \mu \dagger}+\mathcal{P}_{b}^{* \mu} \mathcal{P}_{a}^{\dagger}\right) \partial^{\alpha} \mathbb{V}_{b a}^{\beta} \\
-\sqrt{2} \beta g_{V} \mathcal{P}_{b}^{*} \cdot \mathcal{P}_{a}^{* \dagger} v \cdot \mathbb{V}_{b a} \\
-i 2 \sqrt{2} \lambda g_{V} \mathcal{P}_{b}^{* \mu} \mathcal{P}_{a}^{* \nu \dagger}\left(\partial_{\mu} \mathbb{V}_{\nu}-\partial_{\nu} \mathbb{V}_{\mu}\right)_{b a}
\end{gathered}
$$

with the pseudoscalar mesons fields $\mathcal{P}^{T}=\left(D^{+}, D^{0}\right)$ and vector mesons fields $\mathcal{P}^{* T}=\left(D^{*+}, D^{* 0}\right) . v=(1, \mathbf{0})$. The light pseudoscalar meson matrix $\mathbb{P}$ and the light vector meson matrix $\mathbb{V}_{\mu}$ have the conventional forms of

$$
\begin{aligned}
\mathbb{P} & =\left(\begin{array}{ccc}
\frac{\pi^{0}}{\sqrt{2}}+\frac{\eta}{\sqrt{6}} & \pi^{+} & K^{+} \\
\pi^{-} & -\frac{\pi^{0}}{\sqrt{2}}+\frac{\eta}{\sqrt{6}} & K^{0} \\
K^{-} & \bar{K}^{0} & -\sqrt{\frac{2}{3} \eta}
\end{array}\right), \\
\mathbb{V}_{\mu} & =\left(\begin{array}{ccc}
\frac{\rho^{0}}{\sqrt{2}}+\frac{\omega}{\sqrt{2}} & \rho^{+} & K^{*+} \\
\rho^{-} & -\frac{\rho^{0}}{\sqrt{2}}+\frac{\omega}{\sqrt{2}} & K^{* 0} \\
K^{*-} & \bar{K}^{* 0} & \phi
\end{array}\right)_{\mu},
\end{aligned}
$$

respectively. We employ the coupling constants $g_{s}=0.76$ $[19,20], g=0.59$ [21,22], $f_{\pi}=0.132 \mathrm{GeV}, \beta=0.9$ [21], $\lambda=0.56 \mathrm{GeV}^{-1}$ [21], and $g_{V}=5.8$.

When we consider the isospin breaking effect and the $S-D$ wave mixing effect, the discussed channels for the coupled $D^{0} D^{*+} / D^{+} D^{* 0}$ systems include $D^{0} D^{*+}\left({ }^{3} S_{1}\right)$, $D^{0} D^{*+}\left({ }^{3} D_{1}\right), D^{+} D^{* 0}\left({ }^{3} S_{1}\right)$, and $D^{+} D^{* 0}\left({ }^{3} D_{1}\right)$. The OBE effective potentials for the coupled $D^{0} D^{*+} / D^{+} D^{* 0}$ system with $J^{P}=1^{+}$read as

$$
V=\left(\begin{array}{ll}
\mathcal{V}^{D^{0} D^{*+} \rightarrow D^{0} D^{*+}} & \mathcal{V}^{D^{+} D^{* 0} \rightarrow D^{0} D^{*+}} \\
\mathcal{V}^{D^{0} D^{*+} \rightarrow D^{+} D^{* 0}} & \mathcal{V}^{D^{+} D^{* 0} \rightarrow D^{+} D^{* 0}}
\end{array}\right)
$$

with 


$$
\begin{aligned}
\mathcal{V}^{D^{0} D^{*+} \rightarrow D^{0} D^{*+}}= & -g_{s}^{2} \mathcal{Y}_{\sigma}+\frac{g^{2}}{3 f_{\pi}^{2}} \mathcal{Z}_{\pi 0}^{\prime}-\frac{1}{4} \beta^{2} g_{V}^{2}\left(\mathcal{Y}_{\rho}-\mathcal{Y}_{\omega}\right) \\
& +\frac{2}{3} \lambda^{2} g_{V}^{2} \mathcal{X}_{\rho 0}, \\
\mathcal{V}^{D^{+} D^{* 0} \rightarrow D^{0} D^{*+}}= & -\frac{g^{2}}{6 f_{\pi}^{2}}\left(\mathcal{Z}_{\pi 2}-\frac{1}{3} \mathcal{Z}_{\eta 2}\right)+\frac{1}{2} \beta^{2} g_{V}^{2} \mathcal{Y}_{\rho} \\
& -\frac{1}{3} \lambda^{2} g_{V}^{2}\left(\mathcal{X}_{\rho 2}-\mathcal{X}_{\omega 2}\right),
\end{aligned}
$$

$$
\begin{aligned}
\mathcal{V}^{D^{+} D^{* 0} \rightarrow D^{+} D^{* 0}}= & -g_{s}^{2} \mathcal{Y}_{\sigma}+\frac{g^{2}}{3 f_{\pi}^{2}} \mathcal{Z}_{\pi 1}-\frac{1}{4} \beta^{2} g_{V}^{2}\left(\mathcal{Y}_{\rho}-\mathcal{Y}_{\omega}\right) \\
& +\frac{2}{3} \lambda^{2} g_{V}^{2} \mathcal{X}_{\rho 1} .
\end{aligned}
$$

The variables in the above equations are

$$
\begin{aligned}
q_{0} & =m_{D^{*+}}-m_{D^{0}}, \quad q_{1}=m_{D^{* 0}}-m_{D^{+}}, \\
q_{2} & =\frac{m_{D^{*+}}^{2}+m_{D^{+}}^{2}-m_{D^{0}}^{2}-m_{D^{* 0}}^{2}}{2\left(m_{D^{0}}+m_{D^{*+}}\right)}, \\
\Lambda_{i}^{2} & =\Lambda^{2}-q_{i}^{2}, \quad m_{\rho / \omega i}^{2}=m_{\rho / \omega}^{2}-q_{i}^{2}, \\
m_{\pi 0}^{2} & =q_{0}^{2}-m_{\pi^{+}}^{2}, \quad m_{\pi 1}=m_{\pi^{+}}^{2}-q_{1}^{2}, \\
m_{\pi 2} & =m_{\pi^{0}}^{2}-q_{2}^{2}, \quad m_{\eta 2}=m_{\eta}^{2}-q_{2}^{2} .
\end{aligned}
$$

In the above expressions, we have defined several useful functions,

$$
\begin{aligned}
\mathcal{Y}_{E}= & \mathcal{O}_{1}\left[J^{P}\right] Y\left(\Lambda, m_{E}, r\right) \\
= & \mathcal{O}_{1}\left[J^{P}\right]\left(\frac{e^{-m_{E} r}-e^{-\Lambda^{2} r}}{4 \pi r}-\frac{\Lambda^{2}-m_{E}^{2}}{8 \pi \Lambda} e^{-\Lambda r}\right), \\
\mathcal{Z}_{E i}= & \left(\mathcal{O}_{1}\left[J^{P}\right] \nabla^{2}+\mathcal{O}_{2}\left[J^{P}\right] r \frac{\partial}{\partial r} \frac{1}{r} \frac{\partial}{\partial r}\right) Y\left(\Lambda_{i}, m_{E i}, r\right), \\
\mathcal{Z}_{E i}^{\prime}= & \left(\mathcal{O}_{1}\left[J^{P}\right] \nabla^{2}+\mathcal{O}_{2}\left[J^{P}\right] r \frac{\partial}{\partial r} \frac{1}{r} \frac{\partial}{\partial r}\right) \\
& \times\left(\frac{\cos \left(m_{E i} r\right)-e^{-\Lambda_{i} r}}{4 \pi r}-\frac{\left(\Lambda_{i}^{2}+m_{E i}^{2}\right) \exp \left(-\Lambda_{i} r\right)}{8 \pi \Lambda_{i}}\right), \\
\mathcal{X}_{E i}= & \left(2 \mathcal{O}_{1}\left[J^{P}\right] \nabla^{2}-\mathcal{O}_{2}\left[J^{P}\right] r \frac{\partial}{\partial r} \frac{1}{r} \frac{\partial}{\partial r}\right) Y\left(\Lambda_{i}, m_{E i}, r\right) .
\end{aligned}
$$

Here, $\mathcal{O}_{1}\left[J^{P}\right]$ and $\mathcal{O}_{2}\left[J^{P}\right]$ are the spin-spin interactions $\boldsymbol{\epsilon}_{1} \cdot \boldsymbol{\epsilon}_{3}^{\dagger}, \boldsymbol{\epsilon}_{1} \cdot \boldsymbol{\epsilon}_{4}^{\dagger}$ and the tensor force operators $S\left(\hat{r}, \boldsymbol{\epsilon}_{1}, \boldsymbol{\epsilon}_{3}^{\dagger}\right)$, $S\left(\hat{r}, \boldsymbol{\epsilon}_{1}, \boldsymbol{\epsilon}_{4}^{\dagger}\right) \quad$ with $\quad S(\hat{r}, \boldsymbol{x}, \boldsymbol{y})=3(\hat{r} \cdot \boldsymbol{x})(\hat{r} \cdot \boldsymbol{y})-(\boldsymbol{x} \cdot \boldsymbol{y})$, respectively. In our calculations, these operators are replaced by numerical matrices $\langle f|\mathcal{O}| i\rangle,|i\rangle$ and $\langle f|$ stand for the spin-orbit wave functions for the initial and final states, respectively, i.e.,

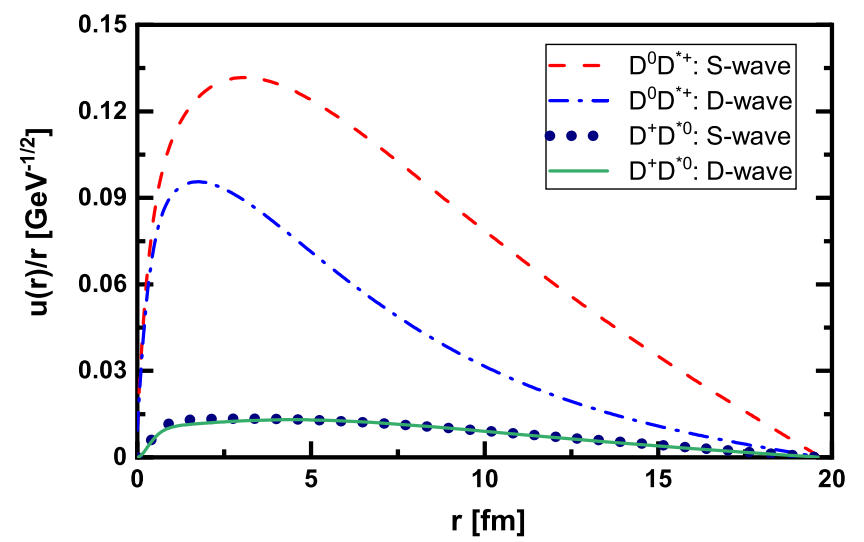

FIG. 1. The radial wave functions for the doubly charmed $D^{0} D^{*+} / D^{+} D^{* 0}$ molecular state with $J^{P}=1^{+}$.

$\begin{aligned} & \boldsymbol{\epsilon}_{1} \cdot \boldsymbol{\epsilon}_{3}^{\dagger} \\ & \boldsymbol{\epsilon}_{1} \cdot \boldsymbol{\epsilon}_{4}^{\dagger}\end{aligned} \mapsto\left(\begin{array}{ll}1 & 0 \\ 0 & 1\end{array}\right), \quad \begin{aligned} & S\left(\hat{r}, \boldsymbol{\epsilon}_{1}, \boldsymbol{\epsilon}_{3}^{\dagger}\right) \\ & S\left(\hat{r}, \boldsymbol{\epsilon}_{1}, \boldsymbol{\epsilon}_{4}^{\dagger}\right)\end{aligned} \mapsto\left(\begin{array}{cc}0 & -\sqrt{2} \\ -\sqrt{2} & 1\end{array}\right)$.

Here, we can also find an approximate relation

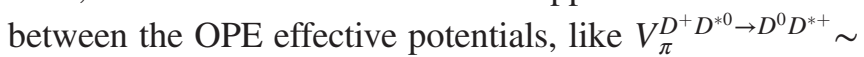
$-2 V_{\pi}^{D^{0} D^{*+} \rightarrow D^{0} D^{*+}}$, where the slight deviation from the exact relation is due to the different exchanged momentum of the exchanged mesons.

We first solve the coupled channel Shrödinger equation and obtain the bound state properties for the coupled $D^{0} D^{*+} / D^{+} D^{* 0}$ system with $J^{P}=1^{+}$. When the cutoff takes a reasonable value $\Lambda=1.16 \mathrm{GeV}$, we find a very shallow bound state with a binding energy $E=-259.90 \mathrm{keV}$. Thus, its mass $M=m_{D^{0}}+m_{D^{*+}}+E$ overlaps with the mass of the $T_{c c}^{+}$. In Fig. 1, we present the radial wave functions for the doubly charmed $D^{0} D^{*+} / D^{+} D^{* 0}$ molecular state with $J^{P}=1^{+}$. It is a typical loosely bound molecular state. Its root-mean-square radius is $6.28 \mathrm{fm}$, which is much larger than the size of its components. The $S$-wave $D^{0} D^{*+}$ and $D^{+} D^{* 0}$ components are dominant. Their probabilities $\int d^{3} r\left|\psi_{i}(r)\right|^{2} / \sum_{i} \int d^{3} r\left|\psi_{i}(r)\right|^{2}$ are $72.52 \%$ and $25.85 \%$, respectively.

We further study the phase shifts for the coupled $D^{0} D^{*+} / D^{+} D^{* 0}$ systems with $J^{P}=1^{+}$to search for the doubly charmed resonant tetraquark state. Here, we adopt the same OBE effective potentials for the coupled $D^{0} D^{*+} / D^{+} D^{* 0}$ systems with $J^{P}=1^{+}$and the same cutoff value $\Lambda=1.16 \mathrm{GeV}$. In general, a typical Breit-Wigner resonance appears in the position with $\delta=(n+1 / 2) \pi$, where the cross section $\sigma(E)$ reaches the maximum $\sigma_{\mathrm{Max}}\left(E_{0}\right)$, and $E_{0}$ corresponds to the mass of the resonance. The width of the resonance reads as $\Gamma=2 /\left(\frac{d \delta(E)}{d E}\right)_{E_{0}}$. As shown in the Fig. 2, there exists a doubly charmed resonance $T_{c c}^{++}$in the phase shift for the $S$-wave $D^{0} D^{*+}$ channel, its mass and width are 


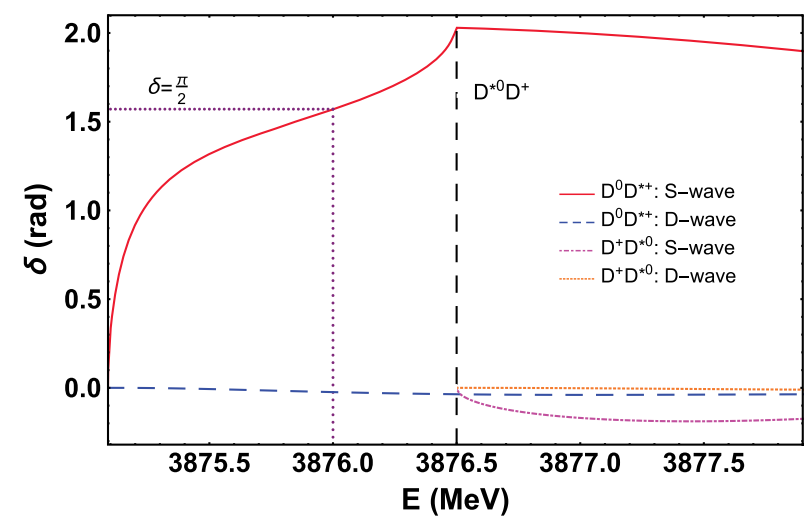

FIG. 2. Phase shifts for the coupled $D^{0} D^{*+} / D^{+} D^{* 0}$ system with $J^{P}=1^{+}$. Here, we adopt the same cutoff value $\Lambda=1.16 \mathrm{GeV}$, the dotted line shows the mass position of the obtained doubly charmed resonance.

$$
m=3876 \mathrm{MeV}, \quad \Gamma=412 \mathrm{keV},
$$

respectively. In fact, the $T_{c c}^{\prime+}$ state is not a shape-type resonance but a Feshbach-type resonance. Once we turn off the contribution from the $D^{+} D^{* 0}$ channel, it disappears. Thus, the isospin breaking effect plays a very important role in forming the $T_{c c}^{++}$state.

To summarize, we have obtained a loosely bound doubly charmed molecular tetraquark and a doubly charmed resonance from the $D^{0} D^{*+} / D^{+} D^{* 0}$ interactions with $J^{P}=1^{+}$. In Fig. 3, we fit the $D^{0} D^{0} \pi^{+}$mass spectrum with our obtained mass for the $T_{c c}^{+}$molecule state and our obtained mass and width for the $T_{c c}^{\prime+}$ resonance. We use the experimental value $\Gamma=410 \mathrm{keV}$ for the decay width of the $T_{c c}^{+}$in the fitting [4]. Our fit is consistent with the experimental data. Therefore, after considering the isospin breaking effect, the $T_{c c}^{+}$state matches the loosely bound doubly charmed molecular tetraquark explanation very well. Since the probabilities ratio for the $S$-wave $D^{0} D^{*+}$ and $D^{+} D^{* 0}$ components is $2.8: 1$, the isospin breaking effect does play an important role in generating this doubly charmed molecular tetraquark. Simultaneously, there exists a doubly charmed resonance $T_{c c}^{++}$between the $D^{0} D^{*+}$ and $D^{+} D^{* 0}$ mass thresholds. These two doubly charmed states are the mixture of the $D^{0} D^{*+}$ and $D^{+} D^{* 0}$ components after considering the isospin breaking effect, which satisfies

$$
\left(\begin{array}{l}
\left|T_{c c}^{+}\right\rangle \\
\left|T_{c c}^{++}\right\rangle
\end{array}\right)=\left(\begin{array}{cc}
\cos \theta & \sin \theta \\
-\sin \theta & \cos \theta
\end{array}\right)\left(\begin{array}{l}
\left|D^{0} D^{*+}\right\rangle \\
\left|D^{+} D^{* 0}\right\rangle
\end{array}\right) .
$$

By using the probability ratio for the $D^{0} D^{*+}$ and $D^{+} D^{* 0}$ components in the $T_{c c}^{+}$state, the mixing angle is $\theta= \pm 30.8^{\circ}$. In this scenario, the $S$-wave $D^{+} D^{* 0}$ channel is the dominant channel for the doubly charmed $T_{c c}^{+}$ resonant tetraquark.

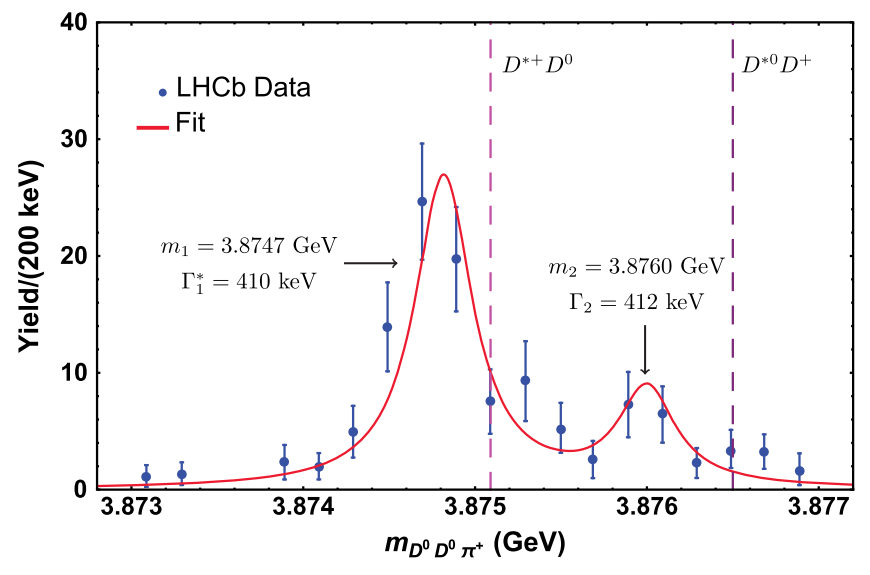

FIG. 3. The fit to the experimental data of the $D^{0} D^{0} \pi^{+}$mass spectrum through the obtained masses and width for the doubly molecule and the doubly resonance. Here, $\Gamma_{1}^{*}=410 \mathrm{keV}$ is the experimental width of the newly $T_{c c}^{+}$[4]. The dash lines label the $D^{0} D^{*+}$ and $D^{+} D^{* 0}$ mass thresholds.

With the same model, the authors of Ref. [6] performed a coupled-channel analysis on the $D^{(*)} D^{(*)}$ system without considering the isospin breaking effects. They found that the OBE interactions from the $D D^{*}$ state with $I\left(J^{P}\right)=$ $0\left(1^{+}\right)$is stronger attractive than those in the isovector state. When we consider the isospin breaking effects, there still exist two bound states, which corresponds to the $T_{c c}$ molecular state and $T_{c c}^{\prime}$ resonance, as shown in the Eq. (11), the probabilities for the $D^{0} D^{*+}$ and $D^{+} D^{* 0}$ components are not the same any more as in the without isospin breaking case.

The decay behavior for the exotic state is very helpful to understand their inner structures. Very recently, several groups discussed the strong and electromagnetic decay behavior for the newly $T_{c c}^{+}$state [23,24]. The strong and electromagnetic decay amplitudes for the $T_{c c}^{+}$and $T_{c c}^{\prime+}$ can be expressed as

$\mathcal{A}_{T_{c c}^{+} \rightarrow D^{0} D^{0} \pi^{+}}=\cos \theta \mathcal{A}_{D^{0} D^{*+} \rightarrow D^{0} D^{0} \pi^{+}}+\sin \theta \mathcal{A}_{D^{+} D^{* 0} \rightarrow D^{0} D^{0} \pi^{+}}$,

$\mathcal{A}_{T_{c c}^{+} \rightarrow D^{+} D^{0} \pi^{0}}=\cos \theta \mathcal{A}_{D^{0} D^{*+} \rightarrow D^{+} D^{0} \pi^{0}}+\sin \theta \mathcal{A}_{D^{+} D^{* 0} \rightarrow D^{+} D^{0} \pi^{0}}$,

$\mathcal{A}_{T_{c c}^{\prime} \rightarrow D^{0} D^{0} \pi^{+}}=-\sin \theta \mathcal{A}_{D^{0} D^{*+} \rightarrow D^{0} D^{0} \pi^{+}}+\cos \theta \mathcal{A}_{D^{+} D^{* 0} \rightarrow D^{0} D^{0} \pi^{+}}$,

$\mathcal{A}_{T_{c c}^{\prime} \rightarrow D^{+} D^{0} \pi^{0}}=-\sin \theta \mathcal{A}_{D^{0} D^{*+} \rightarrow D^{+} D^{0} \pi^{0}}+\cos \theta \mathcal{A}_{D^{+} D^{* 0} \rightarrow D^{+} D^{0} \pi^{0}}$,

$\mathcal{A}_{T_{c c}^{+} \rightarrow D^{0} D^{+} \gamma}=\cos \theta \mathcal{A}_{D^{0} D^{*+} \rightarrow D^{0} D^{+} \gamma}+\sin \theta \mathcal{A}_{D^{+} D^{* 0} \rightarrow D^{0} D^{+} \gamma}$,

$\mathcal{A}_{T_{c c}^{\prime+} \rightarrow D^{0} D^{+} \gamma}=-\sin \theta \mathcal{A}_{D^{0} D^{*+} \rightarrow D^{0} D^{+} \gamma}+\cos \theta \mathcal{A}_{D^{+} D^{* 0} \rightarrow D^{0} D^{+} \gamma}$.

If we only consider the contributions from the tree diagram as shown in Ref. [23,25], we can obtain $\mathcal{R}_{1}=$ $\Gamma\left[T_{c c}^{+} \rightarrow D^{0} D^{0} \pi^{+}\right] / \Gamma\left[T_{c c}^{++} \rightarrow D^{0} D^{0} \pi^{+}\right]=\cos ^{2} \theta: \sin ^{2} \theta=2.80$, which explains why the significance for the $T_{c c}^{\prime+}$ state is a little small compared to the $T_{c c}^{+}$. After neglecting the very small contribution from the $D^{*+} \rightarrow D^{+} \gamma$ process, $\mathcal{R}_{2}=$ $\Gamma\left[T_{c c}^{+} \rightarrow D^{0} D^{+} \gamma\right]: \Gamma\left[T_{c c}^{+} \rightarrow D^{0} D^{+} \gamma\right]=\sin ^{2} \theta: \cos ^{2} \theta=0.35$. 
For the $D^{+} D^{0} \pi^{0}$ final states, the decay width ratio between these two doubly charmed tetraquark is a little complicated due to undetermined partial decay width $\Gamma\left[D^{* 0} \rightarrow D^{0} \pi^{0}\right]$. Assuming that $\Gamma\left[D^{* 0} \rightarrow D^{0} \pi^{0}\right]$ is of the same order or even larger than $\Gamma\left[D^{* 0} \rightarrow D^{0} \pi^{0}\right], \quad \mathcal{R}_{3}=\Gamma\left[T_{c c}^{+} \rightarrow D^{+} D^{0} \pi^{0}\right] /$ $\Gamma\left[T_{c c}^{\prime+} \rightarrow D^{+} D^{0} \pi^{0}\right]$ is less than 0.7. Therefore, the $D^{0} D^{+} \gamma$ and $D^{+} D^{0} \pi^{0}$ decay mode shall be the prime channels to search for the $T_{c c}^{\prime+}$ state.

\section{SUMMARY}

At the European Physical Society conference on high energy physics 2021, the LHCb Collaboration reported the observation of the doubly charmed tetraquark $T_{c c}^{+}$in the $D^{0} D^{0} \pi^{+}$mass spectrum. Stimulated by its very near threshold property, we perform an isospin breaking effect analysis on the $D^{0} D^{*+} / D^{+} D^{* 0}$ interactions. We adopt the OBE model and consider the $S-D$ wave mixing effect. Our results indicate the newly observed $T_{c c}^{+}$is consistent with the $D^{0} D^{*+} / D^{+} D^{* 0}$ doubly charmed molecular tetraquark with $J^{P}=1^{+}$, while the probabilities for the $S$-wave $D^{0} D^{*+}$ and $D^{+} D^{* 0}$ components are $72.51 \%$ and $25.85 \%$, respectively.

Using the same OBE effective potentials, we obtain a coupled $D^{0} D^{*+} / D^{+} D^{* 0}$ doubly charmed resonance $T_{c c}^{+}$, whose mass and decay width are $m=3876 \mathrm{MeV}$, $\Gamma=412 \mathrm{keV}$, respectively. Based on the $T_{c c}^{+}$and $T_{c c}^{+}$ being the mixture of the $D^{0} D^{*+}$ and $D^{+} D^{* 0}$ components, we further discuss their strong and electromagnetic decay properties. Our quantitative analysis indicates that it is a little difficult to identify the predicted $T_{c c}^{\prime+}$ doubly charmed tetraquark in the $D^{0} D^{0} \pi^{+}$. However, it is promising to search for the $T_{c c}^{+}$state in the $D^{0} D^{+} \gamma$ and $D^{+} D^{0} \pi^{0}$ decay modes.

We strongly encourage our experimental colleague to pay more attention to the structure between the $D^{0} D^{*+}$ and $D^{+} D^{* 0}$ thresholds with more precise data. If this substructure can be confirmed in the near future, it shall provide very strong evidence of the existence of the doubly charmed molecules.

\section{ACKNOWLEDGMENTS}

This work is supported by the National Natural Science Foundation of China under Grants No. 11975033 and No. 12070131001, the China National Funds for Distinguished Young Scientists under Grant No. 11825503 , National Key Research and Development Program of China under Contract No. 2020YFA0406400, and the 111 Project under Grant No. B20063, the Fundamental Research Funds for the Central Universities under Grants No. lzujbky-2021-sp24. R. C. is supported by the National Postdoctoral Program for Innovative Talent.
[1] M. Gell-Mann, A schematic model of baryons and mesons, Phys. Lett. 8, 214 (1964).

[2] G. Zweig, An SU(3) model for strong interaction symmetry and its breaking. Version 1, CERN-TH-401.

[3] G. Zweig, An SU(3) model for strong interaction symmetry and its breaking. Version 2, CERN-TH-412.

[4] R. Aaij et al. (LHCb Collaboration), Observation of an exotic narrow doubly charmed tetraquark, arXiv:2109 .01038 .

[5] R. Aaij et al. (LHCb Collaboration), Study of the doubly charmed tetraquark $T_{c c}^{+}$, arXiv:2109.01056.

[6] N. Li, Z. F. Sun, X. Liu, and S. L. Zhu, Coupled-channel analysis of the possible $D^{(*)} D^{(*)}, \bar{B}^{(*)} \bar{B}^{(*)}$ and $D^{(*)} \bar{B}^{(*)}$ molecular states, Phys. Rev. D 88, 114008 (2013).

[7] H. Xu, B. Wang, Z. W. Liu, and X. Liu, DD* potentials in chiral perturbation theory and possible molecular states, Phys. Rev. D 99, 014027 (2019).

[8] N. Li and S. L. Zhu, Isospin breaking, Coupled-channel effects and diagnosis of X(3872), Phys. Rev. D 86, 074022 (2012).

[9] T. Gutsche, M. A. Ivanov, J. G. Korner, and V.E. Lyubovitskij, Isospin-violating strong decays of scalar single-heavy tetraquarks, Phys. Rev. D 94, 094012 (2016).
[10] H. X. Chen, W. Chen, X. Liu, and S. L. Zhu, The hiddencharm pentaquark and tetraquark states, Phys. Rep. 639, 1 (2016).

[11] Y. R. Liu, H. X. Chen, W. Chen, X. Liu, and S. L. Zhu, Pentaquark and Tetraquark states, Prog. Part. Nucl. Phys. 107, 237 (2019).

[12] F. K. Guo, C. Hanhart, U. G. Meißner, Q. Wang, Q. Zhao, and B.S. Zou, Hadronic molecules, Rev. Mod. Phys. 90, 015004 (2018).

[13] T. M. Yan, H. Y. Cheng, C. Y. Cheung, G. L. Lin, Y. C. Lin, and H. L. Yu, Heavy quark symmetry and chiral dynamics, Phys. Rev. D 46, 1148 (1992); Erratum, Phys. Rev. D 55, 5851 (1997).

[14] M. B. Wise, Chiral perturbation theory for hadrons containing a heavy quark, Phys. Rev. D 45, R2188 (1992).

[15] G. Burdman and J. F. Donoghue, Union of chiral and heavy quark symmetries, Phys. Lett. B 280, 287-291 (1992).

[16] R. Casalbuoni, A. Deandrea, N. Di Bartolomeo, R. Gatto, F. Feruglio, and G. Nardulli, Phenomenology of heavy meson chiral Lagrangians, Phys. Rep. 281, 145 (1997).

[17] A. F. Falk and M. E. Luke, Strong decays of excited heavy mesons in chiral perturbation theory, Phys. Lett. B 292, 119 (1992). 
[18] G. J. Ding, Are $Y(4260)$ and $Z_{2}^{+}(4250) D_{1} D$ or $D_{0} D^{*}$ hadronic molecules? Phys. Rev. D 79, 014001 (2009).

[19] R. Machleidt, K. Holinde, and C. Elster, The bonn meson exchange model for the nucleon nucleon interaction, Phys. Rep. 149, 1 (1987).

[20] F. L. Wang, R. Chen, Z. W. Liu, and X. Liu, Probing new types of $P_{c}$ states inspired by the interaction between $S$-wave charmed baryon and anti-charmed meson in a $\bar{T}$ doublet, Phys. Rev. C 101, 025201 (2020).

[21] C. Isola, M. Ladisa, G. Nardulli, and P. Santorelli, Charming penguins in $B \rightarrow K^{*} \pi, K(\rho, \omega, \phi)$ decays, Phys. Rev. D 68 , 114001 (2003).
[22] P. A. Zyla et al. (Particle Data Group), Review of particle physics, Prog. Theor. Exp. Phys. 2020, 083C01 (2020).

[23] L. Meng, G. J. Wang, B. Wang, and S. L. Zhu, Strong and electromagnetic decays in the long-distance to identify the structure of the $T_{c c}^{+}$, Phys. Rev. D 104, L051502 (2021).

[24] X. Z. Ling, M. Z. Liu, L. S. Geng, E. Wang, and J. J. Xie, Can we understand the decay width of the $T_{c c}^{+}$state? arXiv:2108.00947.

[25] N. Li, Z. F. Sun, X. Liu, and S. L. ZHu, Perfect DD* molecular prediction matching the $T_{c c}$ observation at $\mathrm{LHCb}$, Chin. Phys. Lett. 38, 092001 (2021). 